\title{
La Escuela como Contexto de Contención Social y AFECTIVA
}

\author{
School as a Context of Social and Affective Contention
}

\author{
Paula Ascorra Costa \\ Especialista en Psicología Social \\ Pontificia Universidad Católica de Valparaíso \\ Av. El Bosque 1950, Sausalito, Viña del Mar \\ pascorra@ucv.cl
}

Helga Arias Zamora

Pontificia Universidad Católica de Valparaíso Av. El Bosque 1950, Sausalito, Viña del Mar

\section{Catalina Graff Gutiérrez}

Pontificia Universidad Católica de Valparaíso Av. El Bosque 1950, Sausalito, Viña del Mar

Resumen: La Reforma Educacional Chilena, iniciada en los 90, planteó como objetivo mejorar la calidad y equidad de la educación, intentando favorecer los procesos de deuteroaprendizaje, con el objeto de crear sujetos críticos capaces de relacionarse con distintos tipos de conocimiento. Lo anterior supuso introducir un cambio en las relaciones que tradicionalmente se desarrollaban en contextos escolares. Por lo anterior, se hizo necesario analizar las interacciones entre práctica pedagógica y clima de aula, tema que nos proponemos desarrollar en el presente artículo

El estudio se llevó a cabo en un $8^{0}$ año básico de la comuna de Villa Alemana, Chile. Se utilizó el enfoque metodológico cualitativo, a través de estrategias descriptivo-interpretativo. La producción de la información se realizó utilizando la observación de campo y entrevistas grupales e individuales.

La información producida permite sostener que el clima de aula y la práctica pedagógica se influyen mutuamente determinando los límites y las posibilidades de desarrollo de conocimiento. En el caso en estudio, se observó que la Escuela y quienes la componen, funcionan privilegiando un rol que busca compensar las carencias de los alumnos, lo que transforma a la institución educacional en un hogar de acogida para menores más que en una escuela.

Palabras Claves: práctica pedagógica, clima de aula, control disciplinario, control social, contención afectiva.

Abstract: The Chilean Educational Reform, started in the 90s, had as an objective to better the quality and equity of education, trying to favour processes of deuterolearning, with the purpose of creating critical human beings able to get acquainted with different types of knowledge: This required a change in the relations that traditionally are developed pin school contexts. So it was necessary to analyze the interactions between pedagogical practice and room climate, subject we develop in the article.

The study took place in an $8^{\text {th }}$ grade in Villa Alemana, Chile trough a a qualitative descriptive-interactive methodology.

The information was gathered utilizing field observation and individual and group interviews. 
The information obtained leads to the conclusion that room climate and pedagogical practice have mutual influence determining the limits and possibilities of development of knowledge. In this case, the school and those who are a part of it, function emphasizing a rol tending to compensate the students' needs; this transforms the educational institution intra home more than in a school.

Key words: pedagogical practice, room climate, disciplinary control, social control, affective contention

\section{INTRODUCCIÓN}

Producto del avance tecnológico y la globalización de los mercados, se ha planteado que la única forma de lograr el desarrollo es vía educación. Podemos observar que los países desarrollados, presentan sujetos que posee variadas habilidades: manejo de dos o más idiomas, manejo de herramientas computacionales, habilidades de búsqueda y filtraje de información, etc. El mundo actual, ya no es el de la década del 80. Se habla de un nuevo analfabetismo y la necesidad de desarrollar habilidades transversales que garanticen procesos de aprendizaje, en vez de procesos de almacenaje de información. Es así como cobran relevancia a escala mundial los temas de Reforma Educacional -en el ámbito escolar- y educación continua y conversión laboral, en el profesional.

La Reforma Educacional Chilena (García Huidobro, 1999) plantea como objetivo central mejorar en forma sustantiva la educación en cuanto a la equidad ${ }^{1}$ y calidad, intentando "favorecer procesos de aprendizaje que vayan construyendo sujetos críticos y reflexivos, capaces de relacionarse de manera distinta con el conocimiento, con la capacidad de comprender, explicar y criticar su realidad, con la capacidad de buscar y crear caminos, con la posibilidad de ir más allá de los límites impuestos" (Assaél et al., 1993).

Para implementar este tipo de transformaciones, es indispensable hacer visibles los procesos y mecanismos que están en la base de las prácticas pedagógicas y que van más allá de lo explícito, teniendo además un papel preponderante al momento de generar una noción de entendimiento y comprensión de los procesos que están presentes en el contexto del aula.

Por ello, es necesario ir tomando en cuenta aspectos como la realidad de los alumnos, sus capacidades, habilidades y necesidades, y considerando la postura del docente frente a esta realidad y su disposición a ser parte del cambio.

Es así como la presente investigación centra su interés en las interacciones suscitadas entre docente y alumnos/as, y entre estos entre sí en el contexto del aula; con el fin de construir algunas relaciones y reflexiones que puedan dar luces sobre la dinámica que presentan la práctica pedagógica y el clima de aula en un 8o año de enseñanza general básica de una escuela chilena. 


\section{MARCO DE REFERENCIA}

La presente investigación adopta un enfoque constructivista, entendiendo el aprendizaje y la enseñanza como procesos interactivos inseparables. El aprendizaje sería un proceso de construcción de significados y atribución de sentido, cuyo énfasis se encontraría en la relación que mantienen educadores y educandos. Tal como lo sostiene Ibañez (1989: 119), los procesos sociales no se ubican "en las personas, ni tampoco fuera de ellas, sino que se ubican precisamente entre las personas; es decir, en el espacio de significados del que participan y construyen conjuntamente". Por su parte, la enseñanza no es más que la ayuda necesaria para que este proceso se produzca en la dirección deseada (Marchesi y Martín, 1998).

Si la ayuda ofrecida no conecta de alguna forma con los esquemas de conocimiento del alumno, si no es capaz de movilizarlos y activarlos, y a la vez de forzar su reestructuración, no estará cumpliendo efectivamente con su cometido. La condición básica para que la ayuda educativa sea eficaz y pueda actuar como tal, es que esa ayuda se ajuste a la situación y a las características que, en cada momento, presenta la actividad mental constructiva del alumno.

Esta ayuda supone presentarle a los alumnos retos abordables, situaciones nuevas, cuya resolución les plantee la necesidad de movilizar los esquemas de conocimiento que ya poseen, pero retos que no estén totalmente fuera de sus posibilidades, o más exactamente que no estén fuera de las posibilidades que su actividad y la del profesor conjuntamente puedan aportar (Marchesi A., Martín E., 1998).

En este sentido, la actividad del profesor resultará más o menos eficaz en función de que se ajuste a la que el alumno lleva a cabo en cada momento del aprendizaje. La función del docente, desde esta perspectiva, es facilitar la actividad mental de los alumnos que les permita construir nuevos conocimientos a partir de la reconstrucción y reorganización de los que ya poseen.

Para ello se toma en consideración la noción de zona de desarrollo próximo de Vygotsky (en Marchesi y Martín, 1998; Coll, 1990) y por otra parte, el concepto de andamiaje de Bruner (Wood, Bruner y Ross, en Marchesi y Martín, 1998; Coll, 1990). En ambos casos se entiende el aprendizaje como resultado de la interacción del alumno con un agente social más competente, en este caso el profesor, que aporta al alumno; en el marco de la actividad conjunta, la ayuda que éste necesita.

El clima de aula es una dimensión emergente de las relaciones que los alumnos y profesor/res establecen entre sí. Este se constituinía en un proceso recursivo de interacciones entre los actores educacionales; en donde el actuar del alumno y el del profesor se despliega conforme a una serie de 
normas, hábitos comportamentales, rituales, prácticas sociales existentes en el contexto del aula; los cuales -a su vez- son constituyentes de nuevos patrones socioafectivos de acción. Es decir, las interacciones entre los alumnos y entre éstos y el profesor son determinadas por y determinantes del clima de aula.

En este sentido, el clima es una dimensión dinámica de los procesos sociales; sin embargo, en la medida en que se genera acuerdo o se aceptan las distinciones y reglas de funcionamiento que regulan los procesos sociales y éstas se instauran como reglas de uso entre los participantes, el tipo de clima tiende a estabilizarse. Es debido a esta característica de flexibilidad, pero a su vez de estabilidad que ha sido posible investigar la relación que existe entre ambiente de aula y diversas dimensiones sociales.

La posibilidad de que la escuela sea significada por el alumno como una experiencia emocionalmente positiva va a depender en gran medida del ambiente que logren crear los alumnos y los profesores en el contexto educacional. Hay ambientes en los cuales los alumnos se sienten respetados en sus diferencias y falencias, sienten el apoyo y la solidaridad de sus pares y profesores, se siente identificados con un curso y una escuela particular, sienten que lo que aprenden es útil y significativo; todas éstas son características propias de climas de aula positivos. Por el contrario, existen ambientes escolares que producen irritación, estrés, depresión, falta de motivación, apatía por el colegio, temor al castigo y la equivocación; a este conjunto de características del ambiente es a lo que se denomina clima de aula negativo.

De acuerdo a un reciente estudio realizado por la UNESCO (2002), en el cual se buscaba identificar aquellos factores que podrían influir en el aprendizaje de los alumnos, se encontró que el clima que se generaba en el aula era fundamental. Según lo señalado por Cassasus en UNESCO (2002), se demostró que si se suman todos los factores extraescuela, con los materiales, con los recursos humanos y los factores psicológicos, esta suma es inferior a la importancia que tiene el clima emocional lograda en el aula. De acuerdo a este estudio, serían tres los factores que definirían un buen clima en la sala de clases: no violencia, ausencia de perturbaciones para estudiar y buenos amigos. De acuerdo a Cassasus (2002), si se comparan dos colegios en las mismas condiciones, pero con distintos resultados, la respuesta está en la emocionalidad: el que tiene rendimiento negativo significa que sus profesores son negativos, sus alumnos están estresados y hay violencia en la sala de clases.

\section{METODOLOGÍA}

Para llevar a cabo el presente estudio se utilizó el enfoque metodológico cualitativo, utilizando una estrategia interpretativa, la cual permite llevar a 
cabo el estudio de los fenómenos desde la perspectiva de los sujetos, desde lo descriptivo hasta el establecimiento de relaciones e interpretaciones de aquellos aspectos observados, manteniendo el interés por conocer cómo las personas experimentan y significan el mundo social que construyen (Pérez, 1994).

El diseño de investigación es estudio de caso interpretativo cuya unidad de análisis correspondió a un curso de octavo año de Enseñanza Básica, perteneciente a una escuela municipalizada de la comuna de Villa Alemana, Chile. El curso, conformado por 40 alumnos, se investigó durante las horas de la asignatura de Lenguaje y Comunicación que se impartía tres veces a la semana con una duración de una hora y media cada sesión.

Para la producción de información, se trabajó con las técnicas de la observación persistente y la entrevista. Las observaciones de campo se realizaron fundamentalmente en el contexto del aula, también hubo observaciones ocasionales correspondientes a recreos y formaciones de alumnos en el patio.

Las observaciones del aula se realizaron durante diez sesiones de la asignatura de Lenguaje y Comunicación, con una duración de 90 minutos cada una. Durante las observaciones se grabó en cinta magnetofónica y se tomó notas en un cuaderno de campo acerca de aspectos considerados relevantes.

Se realizó una entrevista semiestructurada a la profesora de la asignatura de Lenguaje y Comunicación al finalizar el proceso de observaciones en el aula, la cual tuvo una duración aproximada de 90 minutos. Además se realizó una entrevista grupal a seis alumnos (cuatro hombres y dos mujeres), de tal manera de tener una muestra que representara dos posturas o situaciones distintas respecto del acontecer en el aula. Esta entrevista tuvo una duración aproximada de 90 minutos.

La información obtenida a través de la transcripción de las clases observadas y de las entrevistas fue analizada mediante el análisis de contenido, el cual comprende "un procedimiento destinado a desestabilizar la inteligibilidad inmediata de la superficie textual, mostrando sus aspectos no directamente intuibles y sin embargo, presentes" (Díaz \& Navarro, en Delgado y Gutiérrez, 1999).

\section{RESULTADOS}

Los hallazgos obtenidos en la investigación se presentarán distinguiendo un nivel descriptivo y uno relacional interpretativo. 
En el nivel descriptivo se presentarán las categorías y subcategorías que emergieron del proceso de codificación de la información; las cuales han sido organizadas en el Diagrama de Arbol №1. Como el lector podrá observar, la categorización se estructura en función de dos grandes tópicos; a saber, la práctica pedagógica y el clima social de aula. Cada una de estas categonías y sus subunidades será descrita por medio de una definición conceptual y un ejemplo.

En el nivel relacional interpretativo se presentará y describirá el Esquema Relacional №1, cuyo objeto es articular, en una noción de sentido, las categorías ya presentadas.

DIAGRAMA DE ARBOL $N^{\circ} 1$

Categorización de Práctica Pedagógica y Clima de Aula

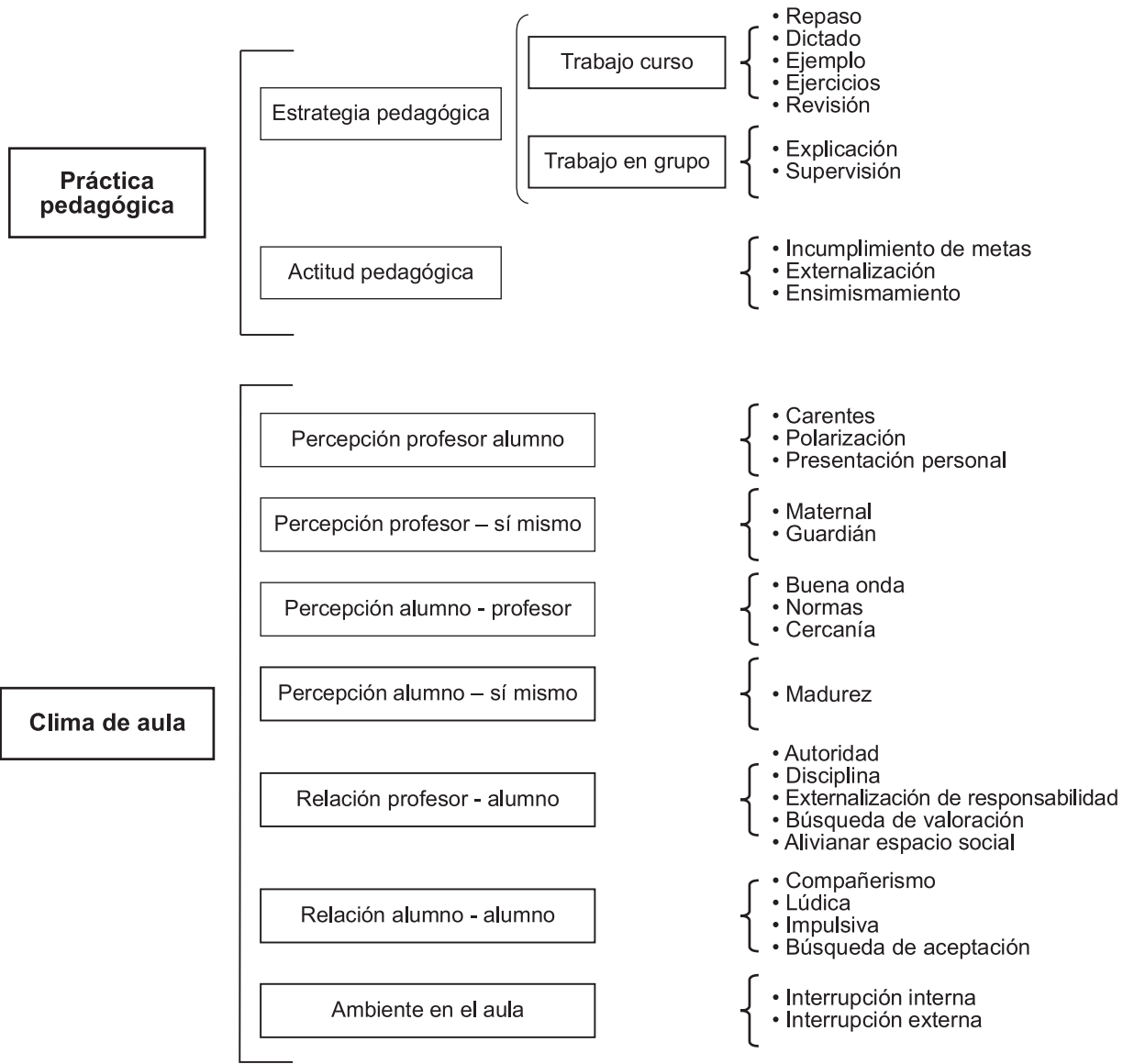

A continuación, se describirán, brevemente, cada uno de las categorías y subcategorías distinguidas. 


\section{PRÁCTICA PEDAGÓGICA}

La práctica pedagógica es el conjunto de acciones y disposiciones que se desarrollan en el contexto del aula durante el proceso de enseñanza y aprendizaje, y que son guiadas por el docente.

Desde esta perspectiva, podemos señalar que la práctica docente observada está conformada por dos grandes categorías, que hemos denominado estrategia pedagógica y actitud pedagógica de la profesora.

\subsection{ESTRATEGIA PEDAGÓGICA}

Las estrategias pedagógicas son todas las actividades voluntarias que realiza la profesora dentro del aula, y que tienen como objetivo la entrega de conocimientos y el aprendizaje de los mismos por parte de los alumnos. Las estrategias pedagógicas de la profesora se presentan en dos modalidades, que son la modalidad trabajo curso y la modalidad trabajo en grupo.

1.1.1 Trabajo en curso: es la realización de actividades con todos los alumnos, se caracterizan por ser un proceso que mantiene un patrón relativamente estable en la realización de las clases.

En primer lugar, se distinguió el repaso que es un estudio ligero que se hace de lo que se tiene visto o estudiado. Éste se manifestó de dos maneras, ya sea preguntando a los alumnos por la materia de la clase anterior; por ejemplo, "'se acuerdan de los complementos?', o bien, realizando la profesora un resumen de lo visto, de tal manera de dar sentido a lo que se quiere exponer a continuación, por ejemplo: "Estabamos viendo el complemento, recordemos que vimos el complemento directo la semana pasada. Bueno, ¿qué son los complementos? Los complementos es todo lo que nos queda en el predicado ¿cierto?, fuera del núcleo todo lo que nos queda son complementos". (Observación en Aula).

En segundo lugar, se distinguió el dictado que es la entrega de los conocimientos teóricos por parte de la profesora para que los alumnos lo vayan escribiendo. Por Ejemplo: "jYa!, El complemento, complemento es una estructura gramatical, es una estructura gramatical, que aporta, que aporta, 0 expresa, 0 expresa distintas circunstancias, distintas circunstancias". (Observación en Aula).

En tercer lugar, se distinguió el ejemplo que corresponde a un caso 0 hecho que sirve de modelo, para que los alumnos vayan comprendiendo la materia. Por ejemplo: "Complemento de modo, ejemplo, ella trabaja con mucha rapidez, ¿cómo trabaja? Con mucha rapidez". (Observación en Aula). 
En cuarto lugar, se distinguió el ejercicio que es un trabajo práctico que sirve de complemento en el aprendizaje de los conocimientos recién entregados. Por ejemplo: "Hagan una actividad ahí, completa las siguientes oraciones con un complemento directo". (Observación en Aula).

En quinto lugar, se distinguió la revisión que es someter una cosa a nuevo examen para corregirla. Esta revisión se realizaba en forma oral a toda la clase, para ello la profesora iba solicitando la participación espontánea de los alumnos o en forma dirigida preguntaba a éstos. Por ejemplo: "¡Ya!, ¿Quién quiere leer sus oraciones para que las vayamos revisando todos juntos?" (Observación en Aula).

1.1.2 Trabajo en Grupo, que es el llevar a cabo actividades que serán realizadas en pequeños grupos de alumnos; se caracteriza por presentar dos etapas fundamentalmente.

En primer lugar, se distinguió la explicación que es la exposición de la materia que permite que sea más comprensible. En ella la profesora enunciaba la actividad y los pasos que debían realizar los alumnos en cada grupo de trabajo.Por ejemplo: "la letra tiene que ser clara, grande, vistosa, acuérdense que ustedes se paran acá adelante y los tienen que ver hasta sus compañeros de atrás cya?". (Observación en Aula).

En segundo lugar, se distinguió la supervisión, que es ejercer la inspección durante el trabajo de los grupos de alumnos. Esto permitía verificar, por una parte, que los alumnos estuvieran realizando el trabajo; y por otra, ir comprobando si se habían comprendido las instrucciones. Por ejemplo: "Ya, cterminaron ustedes?, chicieron la primera actividad?, a verla". (Observación en Aula).

\section{2.- ACTITUD PEDAGÓGICA}

La entendemos como la disposición comunicacional afectiva que manifiesta la profesora, tanto en forma verbal como no verbal, en su interacción con los alumnos durante el proceso de enseñanza y aprendizaje. Creemos que este aspecto es fundamental, ya que es un aspecto inseparable de las estrategias pedagógicas utilizadas por el docente y que como tal, puede ser una herramienta que aporta positivamente al proceso de enseñanza y aprendizaje, o que por el contrario, pueden jugar en contra del mismo.

Es así, como podemos plantear que la actitud pedagógica de la profeso$\mathrm{ra}$, se presenta con una constante que involucra tres aspectos principales, a los que hemos denominado, incumplimiento de metas, externalización de la responsabilidad y ensimismamiento. 
1.2.1.- El Incumplimiento de Metas se caracterizó por todos aquellos planteamientos que propone la profesora a modo de metas, tareas o reglas ante los alumnos y que en reiteradas ocasiones no son cumplidas y se convierten en un reiterado proponer y posponer. Por ejemplo: "Daniela ctrajiste tu trabajo?, Daniela hasta el martes tiene plazo". (Observación en Aula).

1.2.2.- La Externalización de la Responsabilidad se caracterizó por la reiterada alusión a que la responsabilidad de tomar atención, de aprender, de recordar la materia, etcétera, es de los alumnos. Por ejemplo: "...tú habrás salido o qué se yo en ese momento, porque yo pasé la materia"; "...los vimos el año pasado, no se acuerdan pero yo se los pasé". (Observación en Aula).

1.2.3.- El Ensimismamiento incluye todas aquellas actitudes y acciones que señalan el no estar totalmente presente y/o atenta ante diversos aspectos del desarrollo y consecución de la clase, es como si la profesora estuviera preocupada o pendiente de otras cosas, lo que se pudo evidenciar en aspectos como no llevar preparadas las pruebas, interrumpir a los alumnos cuando estaban preguntando a sus compañeros en la disertación, señalar equívocos de alumna y no corregirlos, no indagar en motivos por los cuáles los alumnos no presentan sus trabajos, no recordar si ha entregado o no notas, olvidar pasar ciertos contenidos de la materia, etcétera. Por ejemplo:

P: "Constructores, constructores y obreros trabajan hasta el anochecer, ¿cuántas llevo?" (mientras dicta una prueba)

Ao: "cinco"

(Un Ao pregunta por las notas de la disertación)

P: "iAh! ¿todavía no se las entrego?, mañana las traigo

P: "Se me había olvidado nombrarlo". (Agrega el Complemento de cantidad) (Observación en Aula).

\section{2.- CLIMA DE AULA}

El Clima de aula integra las percepciones de los alumnos/as y docente sobre ellos mismos, sobre los demás (Docente y Alumnos/as) y sobre las interacciones que ocurren en el aula.

Desde esta perspectiva, podemos señalar que presentan como categorías fundamentales, las que tienen relación con las percepciones tanto de la profesora como de los alumnos, respecto de sí mismos, de los demás y de las interacciones que se suscitan en el aula.

2.1.- LAS PERCEPCIONES DE LA PROFESORA SOBRE LOS ALUMNOS son aquellas ideas que posee la profesora sobre las capacidades, actitudes y comportamientos que presentan los alumnos. La percepción de la profesora 
sobre los alumnos se basa fundamentalmente en su visión de personas carentes en diferentes aspectos. En este sentido, los alumnos pertenecerían a familias que son irresponsables y poseedoras de patrones familiares que los alumnos repetirán también en sus propias vidas. Por ejemplo: "Son niñas adolescentes que tienen a sus guaguitas muy chicas y son hijas de mamás muy jóvenes, que les ha sucedido lo mismo. Yo siempre pienso, ponte tú, tener un papá alcohólico y saber lo negativo que es el alcohol, pero ellos repiten la misma historia, no les sirve el ejemplo para no hacerlo ellos, digamos vuelven a caer en lo mismo. Yo sé por ejemplo, que el niñito de la Andrea en unos años más va a ser alumno mío.". (Observación en Aula).

Relacionado con lo anterior, se encuentra la creencia de que los alumnos tienen poca capacidad intelectual. Por ejemplo: "Tienen que ser temas interesantes para que los compañeros tomen atención, no temas aburridos 0 temas difíciles que no van a entender como por ejemplo el cerebro y sus partes, no se van aprender tantos nombres..."; "Qué le vas a pedir a los niños? Si uno tiene que sacarles el mayor provecho.". (Observación en Aula).

Además pudimos distinguir que la profesora posee la percepción de que en el curso se pueden identificar alumnos buenos o malos, en relación, ya sea al comportamiento y/o el rendimiento escolar fundamentalmente. Así es posible hablar de alumnos flojos y alumnos trabajadores, lo cual categorizamos como polarización. Esto tiene como consecuencia, que sea responsabilidad de los propios alumnos identificar quien es flojo y quien es trabajador, y así saber con quien trabajar, estudiar, jugar, etcétera. Por ejemplo: "...no los cuatro que saben más o los cuatro que saben menos...". (Observación en Aula).

La percepción de la profesora sobre la presentación personal de los alumnos/as es relevante, ya que se encuentra presente en la mayoría de la observaciones realizadas, y está en relación a la creencia de que los alumnos deben estar ordenados y limpios, existiendo mayor supervisión sobre el aspecto personal de los hombres. Por ejemplo: "Quiroz colócate la capa antes de salir" (hombre); "Mañana me trae bien cosidos esos pantalones, pónete la capa, todos los días es el cuento contigo, todos los días". (Observación en Aula).

2.2.- PERCEPCIÓN DE LA PROFESORA SOBRE SÍ MISMA categoriza aquellas definiciones de la profesora acerca de sus capacidades, actitudes y comportamiento, y de su interacción con los demás en el contexto esccolar; y que finalmente la llevan a cumplir un determinado "rol" en el aula. Éste se centra básicamente en dos conceptos, el maternal y el guardián.

En el maternal es donde la percepción sobre sí misma se relaciona con el cumplir una función asistencial como madre para los alumnos. Por ejemplo: "...Pero también uno cumple la función, un poco como profesora jefe, 
un poco la función de mamá, o sea el otro día yo fui al hospital, a hablar con la asistente social, con la Andrea (alumna embarazada). 0 sea uno tiene que cumplir con esos roles si no hay apoyo de la familia." (Entrevista profesora).

Y la percepción de cumplir un rol normativo que pone los límites y reglas en el aula, el cual denominaremos guardián. Por ejemplo: "... yo sé que soy bien enérgica con ellos, yo tengo que ser así porque sino no podría controlarlos. De repente me cuestiono un poco, porque a veces son muy pasados para la punta". (Entrevista a la profesora).

\section{3.- PERCEPCIÓN DE LOS ALUMNOS SOBRE LA PROFESORA categoriza} aquellas descripciones que los alumnos hacen acerca de su profesora. Es así como los alumnos perciben a su profesora como "buena onda", basados en las oportunidades que les entrega la profesora en el trabajo en el aula y en la realización de actividades entretenidas. Por ejemplo "Yo la encuentro más que buena onda, porque hay profesoras que dictan, dictan, pero ella se da cuenta que uno ya está como aburrido, achacao, nos da tiempo para descansar. Por ejemplo, nos dicta materia y nos dice 'ya, descasemos 5 minutos, pero no se paren'". (Entrevista alumnos).

Al mismo tiempo, se encuentra presente la percepción de que la profesora, si bien es "bueno" que entregue oportunidades, debe ser exigente con las normas tanto en lo académico como en la disciplina. Por ejemplo: "Lo encontraba bien, en mi otro colegio a una sola señorita le llamábamos "tía", era la que nos daba más confianza. En lo que yo me he fijado, los profesores en el colegio nos han dado muchas posibilidades y mis compañeros se toman mucho eso y no hacen las cosas" (Entrevista alumnos).

Por otra parte se manifiesta un interés por una mayor cercanía en su relación con los alumnos en aspectos afectivos. Por ejemplo: "En el otro colegio eran igual que aquí, sólo que había subdirector, director y eran más estrictos y a la vez también eran más comprensivos". (Entrevista alumnos).

2.4.- PERCEPCIÓN DE LOSALUMNOS SOBRE SÍ MISMOS categoriza aquellas definiciones que poseen los alumnos/as sobre sus capacidades, actitudes y comportamiento, y sobre su interacción con los demás en el contexto escolar. Esta percepción se constituye con las declaraciones que otros hacen acerca de los alumnos. En este caso el tema de la madurez es central en la formación de la identidad. Por ejemplo: "A mi cuesta, todos mis compañeros están como maduros y a mi las profesoras me retan que retan que tengo que madurar un poco más, para la edad que tengo tendría que tener otra mentalidad. Y ahí me he dado cuenta que tengo que madurar no más. Porque la calle no me da de comer. Tengo que estar más junto a mi familia y dedicarme un poco más al colegio". (Entrevista alumnos). 
La "inmadurez" con la cual ellos se perciben sería la causa desde su perspectiva, de los malos resultados obtenidos en distintos aspectos de su desempeño como alumnos/as. "Por ejemplo hacer desorden, igual como alumno, igual yo encuentro que no he madurado tanto de lo que tengo que madurar. Porque yo tengo que ser más maduro" (Entrevista alumnos).

2.5.- RELACIÓN PROFESOR - ALUMNOS es la interacción que ocurre en el aula durante el horario de clases entre la profesora y los alumnos.

Esta relación, se caracteriza por ser jerárquica, en la cual la profesora marca las pautas a seguir en todo momento, imponiendo su autoridad a través de diferentes acciones como por ejemplo, el dar órdenes y mandatos que deben ser acatados por los alumnos, imposibilitando la participación de éstos en las decisiones, ya que la autoridad en la sala de clases es la profesora. Por ejemplo: "Oye, el timbre, yo siempre les he dicho que me avisa a mí, no a ustedes, así que seguimos no más". (Observación en Aula).

En cuanto a la disciplina, la profesora utiliza diferentes estrategias para conseguirla, ya sea a través de llamados de atención tanto verbales como físicos, por ejemplo: "...cállate ¿quieres?/Toma a una alumna como del pelo o la oreja/ no ha hecho nada, nada, nada, puro blablabla". (Observación en Aula).

Como también, a través de amenazas de castigos relacionados con aspectos académicos, como el bajar las notas. Por ejemplo: "A ver, ¡Oyyyy! Que es porfia' la Javiera, Javiera ya te llevo dos cruces pa' bajarte puntos, como tú no entiendes". Ante estas situaciones los alumnos responden de manera pasiva, acatando las órdenes o castigos. (Observación en Aula).

Al mismo tiempo, existe una actitud de la profesora que se caracteriza por la externalización de la responsabilidad frente a los problemas 0 equívocos que ocurran en el aula, ya sea de disciplina o de relación entre alumnos y apoderados. Por ejemplo: "... no somos guardias, no es una guardería... religión nadie quiere entrar... Jimi vaya... salen... si no quieren ir a la escuela, sean sinceros con sus papás, yo no quiero ir, pero no me vengan a molestar... no van a tener licenciatura, sino pagan las fotocopias... a algunos no les interesa, pero a mi me da pena por los que quieren... si ustedes no se hacen respetar... son señoritas... Jimi le tiró una piedra a Javiera, sé que ella no se porta muy bien...". (Observación en Aula)

La relación entre la profesora y los alumnos se caracteriza además por la búsqueda de valoración que tratan de alcanzar los alumnos en la relación con ésta. Por ejemplo: Un alumno pide que le revise los ejercicios, la profesora lo hace, una vez revisado el alumno /sonníe/ y se va a su puesto 
señalando a sus compañeros/as que tiene todo bueno. (Observación en Aula).

2.6.- RELACIÓN ENTRE LOS ALUMNOS es la interacción que ocurre en el aula durante el horario de clases entre los alumnos. Esta relación se caracteriza por el compañerismo, lo cual implica ser cercanos y apoyarse entre ellos, manifestando interés por ejemplo en las actividades que realizan los demás.

Ao: "Juégatela, juégatela no más Mauricio" (Los alumnos aplauden)

Ao: "Bravo!"

Ao: "Un siete"

Ao: "Eso te pasa por pasarte en la calle poh!"

A0: "10 puntos para Mauricio"

(Los Aos que rodean a Mauricio le conversan sobre por qué no lo hizo)

Ao: "Mauricio, no te pongai nervioso, te voy a hacer..." (al alumno que va a disertar)

Ao: "Yo te ayudo a sujetar el papel".

(Observación en Aula)

Además los alumnos mantienen una relación lúdica, caracterizada generalmente por juegos de golpes, manotazos y cosquillas, sin llegar a pelear entre ellos. Por ejemplo: "Ya, el Araneda que tiene tantas ganas de hablar..." (los compañeros que lo rodean le pegan en la cabeza al momento de ser nombrado). (Observación en Aula)

Al mismo tiempo, la relación entre los alumnos se caracteriza por ser en ciertas ocasiones impulsiva, lo cual implica reacciones instantáneas sin mediar reflexión aparentemente. Por ejemplo: "A vo' te voy a pegar afuera cabezón". (Observación en Aula)

2.7.- AMBIENTE EN EL AULA es la situación general del desarrollo del trabajo en el aula. Este se caracterizaba por ser continuamente interrumpido, lo cual dificultaba la continuidad de la dinámica académica.

Las interrupciones internas se producían por ejemplo, cuando la profesora y/o los alumnos salían de la sala de clases sin pedir permiso o cuando llegaban alumnos atrasados a la clase. (Observación en Aula)

Las interrupciones externas se producían cuando ingresaban a la sala de clases personas ajenas al curso, como por ejemplo, apoderados, Jefe U.T.P. o hermanos de alumnos y también cuando se colocaba música ambiental en la sala. (Observación en Aula)

Esto dificultaba y perturbaba la concentración tanto de la profesora como de los alumnos, así como también el desarrollo de la clase. 


\section{ANÁLISIS}

Las sub categorías obtenidas permitieron construir un esquema (Esquema Relacional $\mathrm{N}^{\circ} 1$ ) en el cual se integran las categonías generales y sus relaciones:

\section{Esquema Relacional №1}

Relaciones establecidas entre las categorías emergentes del estudio.

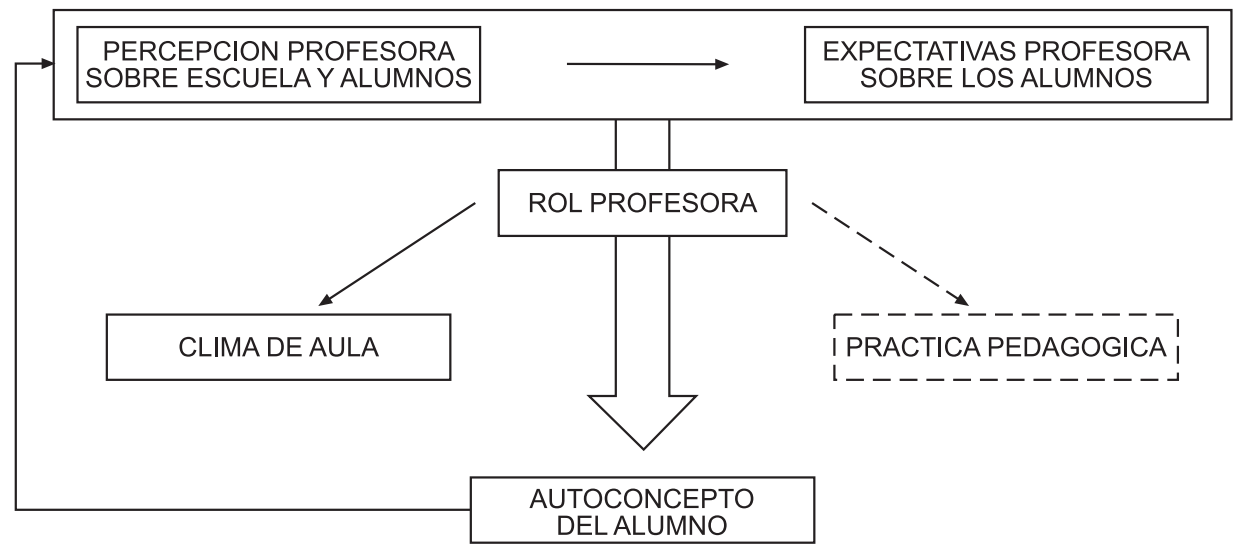

La forma en la cual se va construyendo la práctica pedagógica y el clima de aula, se encuentra en estrecha relación con las percepciones y expectativas que posee la profesora acerca de la escuela y acerca de los alumnos, las cuales se basan fundamentalmente en el contexto sociocultural en el que éstos se encuentran inmersos. Tales percepciones inciden en la construcción que realiza la profesora acerca de su rol como pedagoga. La percepción que posee sobre los alumnos está referida principalmente al nivel socioeconómico, familiar y social de la comunidad a la cual pertenecen. Esto se traduce en definitiva, en percibir al alumno y su familia, como carentes, desvalidos y poco capaces de afrontar diferentes situaciones.

Es así como la profesora asume, para poder dar solución a lo anterior, un rol que se encuentra orientado más al aspecto asistencial que al pedagógico. En este sentido cobran fuerzas las formas de relación que establece con los alumnos en el aula, privilegiando el conseguir que los alumnos logren objetivos relacionados con la formación de hábitos, valores y normas fundamentalmente. Las percepciones que tiene la profesora respecto de los alumnos, determinan en gran parte las expectativas que posee respecto de las capacidades, habilidades y logros de los alumnos, tanto en su desempeño académico actual como en el laboral a futuro. Esto implica, según la profesora, que dada las características que poseería el alumno, es justificable que se le entreguen más facilidades para el cumplimiento de los logros escolares. 
Las percepciones y expectativas que posee la profesora sobre los alumnos, estarían constriñendo el nivel de logros que éstos podrían alcanzar. Se produce así un círculo interactivo en donde prácticas de la profesora y aquellas emanadas desde los alumnos se retroalimentanían mutuamente en función de una concepción de carencia e incapacidad presentada en los sujetos alumnos. Vemos que la profesora limita el desarrollo de las capacidades de sus estudiantes, al exigirles académicamente el cumplimiento de los niveles mínimos de aprendizaje; lo que contribuye a que el alumno se forje un autoconcepto en el cual él "es visto" como poco capaz, o como ellos mismos señalan "inmaduros". Es en este sentido que ambas prácticas sociales perpetúan un proceso recursivo que posiciona, inevitablemente al alumno, en un lugar de dependencia y vulnerabilidad.

En este sentido, podemos señalar que en el ámbito de la práctica pedagógica de la profesora se privilegia el desarrollo de estrategias estructuradas, poco flexibles y directivas, donde se presentan pocos espacios para la participación activa de los alumnos y para el desarrollo de la creatividad de los mismos, y donde se espera que el alumno permanezca callado y tranquilo y hable sólo cuando se le pregunta algo

\section{DISCUSIÓN FINAL}

Tomando en cuenta las categorías obtenidas en la investigación, y relacionando éstas con el caso estudiado, es que se llegó a las siguientes conclusiones:

En primer lugar, el contexto sociocultural en el cual se encuentra inmersa la escuela, corresponde a un sector popular que ha sido socialmente estigmatizado como pobre y marginal. Tal definición es asimilada por la escuela y el cuerpo docente como una realidad que está ahí y que como tal tiene escasas o nulas posibilidades de cambio.

Esta realidad, que sin duda existe, conlleva a que institucionalmente a nivel macro se haga una construcción previa acerca de los profesores que van a ejercer su profesión en dicho lugar. Esta pre-definición institucional, influye en las propias construcciones que realiza la profesora acerca de la escuela a la cual se incorporará a trabajar, de los alumnos con los cuales trabajará y de las familias de las cuales provienen los alumnos.

Como señala Rogers (en Barca, et als 1996), entre los elementos que pueden jugar un papel en la formación de las representaciones y expectativas iniciales del profesor respecto a los alumnos están las opiniones estereotipadas sobre determinadas características de edad, sexo, raza y clase social, entre otras. Esto puede teñir desde un comienzo la relación que se 
produce entre profesor y alumno, y dar pie a determinadas expectativas respecto de la interacción entre ambos.

Esta pre-definición externa sumado a las propias construcciones que realiza la profesora van conformando la percepción de la profesora acerca del propio rol como pedagoga, el cual se irá moldeando paulatinamente a partir de la incorporación a la escuela, el encuentro con la realidad y la asimilación de una cultura escolar propia e independiente de otras escuelas.

En este sentido, la misión que tenga la institución escolar será fundamental en el ejercicio profesional de la profesora, ya que las acciones que realice serán guiadas principalmente por ésta, siempre y cuando se esté en acuerdo con ella. Es importante rescatar que esta misión puede presentarse de dos maneras, una en forma explícita y otra en forma implícita, siendo éste último en muchas ocasiones más determinante que el que está expresado de manera explícita.

Lo que va a dar peso a la misión de la escuela va a ser el hecho de que ésta sea aceptada y compartida por los integrantes de esa colectividad, conformando de esta manera una cultura de la institución escolar que se va a ir perpetuando y consolidando paulatinamente a través del tiempo.

En segundo lugar, la percepción que tiene la profesora acerca de su rol, corresponde a un rol que está abocado a suplir carencias del alumno, principalmente, en el ámbito familiar. En este sentido, su rol representa el mundo maternal, asumiendo diversas responsabilidades extraescolares, en perjuicio de sus funciones pedagógicas. Este rol maternal - asistencial consolida una forma de relación "madre e hijo", donde la profesora asume una postura de quien cuida al hijo y le entrega lo fundamental para su formación social. Es por ello que además su rol incluye la entrega de normas y la preocupación por la presentación personal de los alumnos. En este sentido, los aspectos relacionados con el rol de pedagoga pasan a un segundo nivel de relevancia, dado que éstos tienen menos prioridad para el sentido de formación de los alumnos.

En tercer lugar, la identidad de los alumnos se va conformando en respuesta a los aspectos antes mencionados, la cual tiene como eje central la idea de "carencia", referida a diferentes situaciones en las cuales los alumnos se perciben continuamente en "falta".

En este sentido, y dada las características que posee el medio sociocultural al cual pertenecen los alumnos, podemos hablar de desesperanza aprendi$\mathrm{da}$, es decir, existe la percepción desde el docente de que los hechos que afectan al alumno (nivel socioeconómico, pobreza, marginación), son en cierta medida independientes de su comportamiento o de los esfuerzos que hagan para superarse, y que estas condiciones generalmente traerán consigo características en los alumnos tales como déficit cognitivo y emocional. 
Esto, a su vez, lleva a los alumnos a presentar falta de iniciativa, de persistencia y de comportamiento de logro en general. Para "solucionar" esto, y dada la percepción de incontrolabilidad acerca de su condición, es que se presentan hacia los alumnos reacciones de compasión y simpatía, con la consiguiente ayuda y cooperación hacia su situación.

Todo lo anterior puede conllevar a que los alumnos que se vean enfrentados al fracaso no se comprometan, ni persistan en acciones de logro que los podrían llevar a mejorar su situación.

Esto va constituyendo a un alumno que responde al sistema con un rol pasivo, donde siempre va a demandar ayuda de otro, sin lograr la autovalía y que, por sobre todo, no tendrá la confianza suficiente en sí mismo como para afrontar diversas situaciones.

Como señalan Aron y Milicic (1999), la formación de la propia imagen en el niño, parte de la imagen que los adultos significativos tienen de él. Este es un punto importante para los educadores, quienes con sus comentarios acerca de los éxitos o fracasos del niño, los métodos que se utilizan para corregirlo, los sobrenombres que le ponen, el afecto o rechazo con que se enfrentan a él, van determinando la imagen que el niño se va forjando de sí mismo.

De acuerdo a lo anterior, creemos que el aspecto principal del caso estudiado, es el hecho de que la institución escolar y quienes la componen funcionan de acuerdo a un rol que no respondería a lo netamente educativo, sino más bien a un rol institucional que busca compensar las necesidades sociales más urgentes de la población beneficiaria.

En este sentido la escuela bajo estudio, estaría funcionando como un hogar de acogida, donde su misión fundamental sería entregar alimentación y ser un lugar de permanencia diurna para los alumnos mientras los padres trabajan. Este rol asumido por los diferentes actores del sistema escolar permite comprender el que esta escuela, a través de sus diferentes actividades, no esté priorizando claramente el desarrollo y crecimiento de los alumnos. Esto no estaría permitiendo el proceso de transformación que propone la Reforma Educacional, en el sentido de ir avanzando en la modificación de las orientaciones existentes en las escuelas, en pro de garantizar que los aprendizajes que ocurran fomenten las competencias intelectuales y morales que los alumnos requieren para vivir en la sociedad (García-Huidobro, 1999).

Incluso en el contexto físico de la escuela, existen distintos aspectos que dan cuenta de una realidad donde las cosas son, pero no son; esto lo observamos en el hecho de que las mismas salas u oficinas de la escuela están enunciadas con un letrero que indica a qué corresponde cada una; sin em- 
bargo, sólo dos o tres salas corresponden efectivamente a lo que enuncian, así encontramos el caso por ejemplo de la sala de profesores que está enunciada como Dirección, en tanto la Dirección está enunciada como Educación Diferencial.

De acuerdo a los estudios realizados por Rutter (en Barca et al., 1996), la organización, el proyecto educativo y curricular, plan de estudio y dinámica peculiar de cada centro educativo ejercen un influjo importante en los comportamientos de las aulas, y por lo tanto, de los alumnos.

Por otra parte tampoco se estaría produciendo la entrega de herramientas que permitiesen transformar a futuro la situación sociocultural de los alumnos, sino más bien se presenta una dinámica escolar que estaría apuntando al reproduccionismo social, es decir, la escuela tendenía a reproducir las relaciones sociales de dominación vigentes, donde existen diferentes clases sociales y diferentes oportunidades para cada una de éstas.

En este sentido, las reflexiones en torno a la educación, compartidas por Marx, Lenin, Gramsci y Althusser (citadas en Buenfil, 1991), muestran que las prácticas educativas juegan un papel fundamental en la constitución de los sujetos sociales. De acuerdo a estos autores, la educación es considerada como una práctica social que interviene en forma determinante y específica en dos sentidos: por una parte como conformadora del sujeto revolucionario (a excepción del caso de Althusser) en la medida en que incide en la constitución de una conciencia crítica, constructiva y transformadora; y por otra parte conformadora del sujeto que reproduce y acepta las relaciones sociales dominantes. Este sujeto social acrítico se constituye mediante diversas prácticas sociales, entre las cuales la educativa es ponderada, en el sentido de que su manera de pensar y de actuar, así como los conocimientos acordes a la formación social dominante, son adquiridos y reforzados por las prácticas educativas.

Creemos que de esta forma se va conformando un sistema, en el cual tanto profesora como alumnos cumplen roles que responden funcionalmente a sus propias percepciones, perpetuando y manteniendo los distintos aspectos antes mencionados.

\section{NOTAS}

1 Entendiendo la equidad como una igualdad de oportunidades, se presenta como una incoherencia el hecho de que si bien el Estado está asegurando la igualdad de oportunidades en el acceso a la educación, existe una clara desigualdad en la calidad de la educación que los niños reciben, dependiendo de su nivel socioeconómico; además de esto, y teniendo en cuenta el modelo socioeconómico neoliberal actual, el hecho de haber accedido a la educación (básica y media) y haber finalizado este proceso, actualmente no asegura el acceso inmediato a un empleo y/o a la educación superior, por lo tanto, en este sentido tampoco existiría una igualdad de oportunidades. 


\section{REFERENCIAS BIBLIOGRÁFICAS}

Aron, A y N. Milicic, 1999

Assael, J, I. Guzmán y M.E. Contreras. 1993

Barca, A, J. González, R. González y J. Escoriza, 1996

Buenfil, R. 1991

Coll, C. 1990

Delgado, J y J. Gutiérrez, 1999

García Huidobro, J. 1999

Ibañez, J. 1989

Marchesi, A y E. Martín, 1998

Pérez Serrano, G. 1994

UNESCO. 2002

Woods, P. 1989

Woods, P. 1998
Clima social escolar y desarrollo personal. Un programa de mejoramiento. Andrés Bello, Santiago de Chile, $202 \mathrm{pp}$.

Qué entendemos por calidad de la educación. En: Boletín №18, TED. Programa Interdisciplinario de Investigación en Educación. PIIE. Santiago, 4 pp.

Componentes contextuales y relacionales de la enseñanza. En: Psicología de la instrucción. Vol 3. EUB. Barcelona, 638 pp.

Análisis de discurso y educación. DIE, 182 pp.

Aprendizaje escolar y construcción del conocimiento. Paidós. Barcelona.

Métodos y técnicas cualitativas de investigación en ciencias sociales. Síntesis. Madrid, 206 pp.

La reforma educacional chilena. Popular. España, 335 pp.

Más allá de la sociología: el grupo de discusión técnica y crítica. Sigloveintiuno: Madrid, 428 pp.

Calidad de la enseñanza en tiempos de cambio. Alianza. Madrid, 498 pp.

Técnicas y análisis de datos. En: Investigación Cualitativa. Retos e interrogantes II. La Muralla. Madrid, $198 \mathrm{pp}$.

Resultados escolares en América Latina. Disponible en http://www.minedu.gob.pe/gestion institucional/ ofplanmedumc/indicadores/medicion/ LatinReportWillms\&somers.pdf www.minedu.gob.pe/ gestion institucional/ofplanmedumc/indicadores/ mediciōn/LatinReportWillms\&somers.pdf.

La escuela por dentro. Paidós. Barcelona, 220 pp.

Investigar el arte de la enseñanza: el uso de la etnografía en la educación. Paidós. Barcelona, 232 pp. 